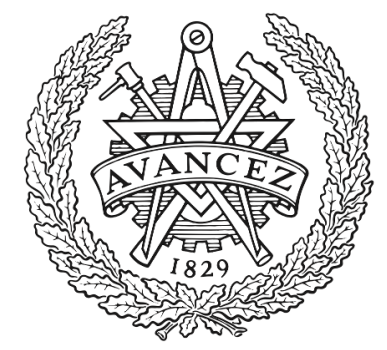

CHALMERS

UNIVERSITY OF TECHNOLOGY

\title{
Pilot Distributions for Phase Tracking in Space-Division Multiplexed Systems
}

Downloaded from: https://research.chalmers.se, 2023-04-26 14:01 UTC

Citation for the original published paper (version of record):

Alfredsson, A., Agrell, E., Wymeersch, H. et al (2017). Pilot Distributions for Phase Tracking in Space-Division Multiplexed Systems. European Conference on Optical Communication, ECOC. http://dx.doi.org/10.1109/ECOC.2017.8345931

N.B. When citing this work, cite the original published paper. 


\title{
Pilot Distributions for Phase Tracking in Space-Division Multiplexed Systems
}

\author{
Arni F. Alfredsson(1), Erik Agrell(1), Henk Wymeersch(1), and Magnus Karlsson(2)
}

(1) Dept. of Electrical Engineering, Chalmers University of Technology, Sweden, $\bowtie$ arnia@chalmers.se

(2) Dept. of Microtechnology and Nanoscience, Chalmers University of Technology, Sweden

\begin{abstract}
Several pilot distributions are compared for pilot-aided joint-core phase tracking in spacedivision multiplexed transmission affected by correlated phase noise. Results show that the best choice of distribution can reduce the bit error rate by a factor up to 170 .
\end{abstract}

\section{Introduction}

Space-division multiplexing (SDM) has gained a significant attention in the past few years. It is believed that this technology may enable substantial throughput increase through the use of fibers with multiple cores and modes, as well as the integration of system components. To further increase spectral efficiency, focus has shifted towards multilevel modulation formats. Using these formats results in more sensitivity to various impairments, which puts more stringent requirements on the performance of compensation methods.

Phase noise in particular can severely diminish performance as the modulation order grows. Assuming SDM transmission using multicore fibers (MCFs) or bundles of fibers, the phase noise will be correlated between spatial channels when they share a common transmitter laser and local oscillator (LO). This correlation may be exploited to either lower the required computational complexity for phase tracking ${ }^{1}$ or to improve the phase-tracking performance, for instance through joint-core processing using pilots ${ }^{2}$. However, the placement of pilot symbols can have a considerable impact on performance. While the general problem of pilot placements has been studied ${ }^{3-5}$, it has not been addressed in the context of phase tracking for SDM transmission.

In this paper, we focus on this problem and compare several types of pilot distributions in terms of the resulting phase-tracking performance. Moreover, we show that the choice of distribution can impact the resulting bit error rate by orders of magnitude, and provide guidelines for how to optimize the pilot placement, which depends on the phase-noise correlation across the spatial channels.

\section{System Model}

Consider an uncoded single-carrier polarizationmultiplexed (PM) transmission in $D$ cores using an MCF or a bundle of fibers, comprising a total of $2 D$ channels. The transmitted symbol block in each channel is modelled as a random vector of length $N$, where every element is drawn uniformly from a set of constellation points. The points are normalized such that their average energy is $E_{\mathrm{s}}$. Certain symbols are designated as pilots, which are known to the receiver.

Furthermore, assume negligible nonlinear distortion and that all signal impairments have been ideally compensated for, with the exception of phase noise and amplified spontaneous emission noise. All channels are considered to share the same light source and $L O$, and thus have correlated phase noise. Assuming one sample per symbol, the resulting discrete-time baseband complex model is expressed as

$$
r_{w, k}^{(i)}=s_{w, k}^{(i)} e^{j \gamma_{w, k}^{(i)}}+n_{w, k}^{(i)}
$$

where $r_{w, k}^{(i)}, s_{w, k}^{(i)}, \gamma_{w, k}^{(i)}$, and $n_{w, k}^{(i)}$ are the received sample, transmitted symbol, total phase noise, and additive white Gaussian noise, respectively, at time $k$ on polarization $w$ in core $i$, for $k=$ $0, \ldots, N-1, w \in\{\mathrm{x}, \mathrm{y}\}$, and $i=0, \ldots, D-1$. Furthermore,

$$
\gamma_{w, k}^{(i)}=\theta_{k}+\delta_{k}^{(i)}+\varepsilon_{w, k}^{(i)},
$$

where $\theta_{k}$ is the laser phase noise (LPN), $\delta_{k}^{(i)}$ is the core-dependent phase drift, and $\varepsilon_{w, k}^{(i)}$ is the polarization-dependent phase drift. All variables on the right-hand side of (2) are statistically independent of each other and are modelled as Gaussian random walks, i.e.,

$$
\begin{gathered}
\theta_{k}=\theta_{k-1}+\Delta_{k}^{\theta}, \\
\delta_{k}^{(i)}=\delta_{k-1}^{(i)}+\Delta_{k}^{\delta}, \\
\varepsilon_{w, k}^{(i)}=\varepsilon_{w, k-1}^{(i)}+\Delta_{k}^{\varepsilon},
\end{gathered}
$$



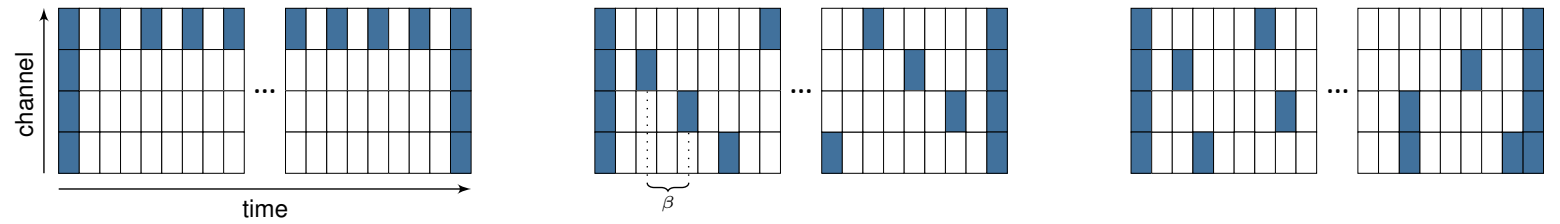

Fig. 1: Illustration of different pilot distributions for $D=2$ cores ( 4 spatial channels) with $p_{\mathrm{a}}=1 / 8$, namely Dense-Sparse (DS) with $\alpha=0$ (left), Wrapped Diagonal (WD) with $\beta=2$ (middle), and a realization of Random (RND) (right). Dark and white blocks correspond to pilot and data symbols, respectively.

where $\theta_{0}, \delta_{0}^{(i)}$, and $\varepsilon_{w, 0}^{(i)}$ are uniformly distributed in the interval $[0,2 \pi)$. Moreover, $\Delta_{k}^{\theta}, \Delta_{k}^{\delta}$, and $\Delta_{k}^{\varepsilon}$ are independent zero-mean Gaussian random variables with variances $\sigma_{\theta}^{2}, \sigma_{\delta}^{2}$, and $\sigma_{\varepsilon}^{2}$, respectively. These variances govern the speed of their corresponding drifts. The variance of the LPN is typically given as $\sigma_{\theta}^{2}=2 \pi \Delta \nu T_{\mathrm{S}}$, where $\Delta \nu$ is the total laser linewidth and $T_{\mathrm{s}}$ is the symbol duration. The core and polarization phase drifts are assumed to drift slower than the LPN, resulting in significant correlation in the total phase noise across all channels ${ }^{6}$.

\section{Phase Noise Compensation}

With an emphasis on high phase-noise tolerance, we have developed several pilot-aided algorithms for the system model in (1), one of which has been proposed ${ }^{2}$. The algorithms perform phase tracking jointly for all channels, taking into account both the common LPN and the relative phase drifts between the channels. As they are pilotaided, their performance is dependent on the pilot distribution, i.e., the manner in which pilot symbols are placed throughout the transmitted symbol block in each channel. In this paper, the algorithm that exhibits the highest phase-noise tolerance will be used for evaluating the pilot distributions.

\section{Pilot Distributions}

Let $p_{\mathrm{a}} \in[0,1]$ be the average pilot rate across all channels. Three types of pilot distributions are compared in terms of the resulting phasenoise tolerance. Types 1 and 2 are restricted to $p_{\mathrm{a}} \leq 1 /(2 D)$, whereas type 3 works for all $p_{\mathrm{a}}$. Regardless of the chosen distribution, pilots are placed at the beginning and the end of the transmitted block in each channel. This improves the phase-tracking performance and has negligible impact on $p_{\mathrm{a}}$ for moderately large block lengths $(N \geq 10000)$.

The first distribution type is parameterized in terms of $\alpha \in[0,1]$ as follows. One channel is selected such that it has a greater or equal pilot rate than the other channels. More specifically, the selected channel has pilot rate $p_{\mathrm{a}}(2 D-(2 D-1) \alpha)$, whereas each of the remaining $2 D-1$ channels has pilot rate $p_{\mathrm{a}} \alpha$. Each channel has its pilots distributed uniformly in the transmitted block. As this type is characterized by dense and sparse pilot placements, it will be referred to as DenseSparse (DS).

The second type is referred to as wrapped diagonal (WD). For notational brevity, denote a channel on polarization $w$ in core $i$ with $(i, w)$ and let $\left(l_{0}, l_{1}, \ldots, l_{2 D-2}, l_{2 D-1}\right)=$ $[(0, \mathrm{x}),(0, \mathrm{y}), \ldots,(D, \mathrm{x}),(D, \mathrm{y})]$. The pilots occur at time indices $\{0, \beta, 2 \beta, \ldots, K \beta\}$, where $K \beta<N$ and $K, \beta$ are nonzero integers. Moreover, if pilot $k$ occurs at channel $l_{i}$, where $i \in\{0,1, \ldots, 2 D-1\}$, then pilot $k+1$ is placed in channel $l_{i+1} \bmod 2 D$.

The third distribution type, referred to as Random (RND), is identified by complete randomness in the pilot placements. Hence, each symbol slot has probability $p_{\mathrm{a}}$ of containing a pilot. This type is of limited practicality but it serves as an interesting benchmark for DS and WD. Fig. 1 depicts examples of the distributions. It should be noted that DS and RND have the advantage of being more easily adaptable to a specific $p_{\mathrm{a}}$ than WD.

\section{Performance Analysis}

Uncoded transmission of PM-64-QAM is simulated through Monte Carlo using the system model in (1), for blocks of up to $N=10000$ symbols per channel and $D=19$ cores, resulting in 38 spatial channels. Bits are Gray encoded before being mapped to symbols. The signal-to-noise ratio per information bit is fixed such that the bit error rate $(B E R)$ for phase-noise free transmission in the absence of pilots is $10^{-3}$. Finally, the core and polarization phase drift speeds are assumed to be equal, i.e., $\sigma_{\delta}^{2}=\sigma_{\varepsilon}^{2} \triangleq \sigma_{\mathrm{r}}^{2}$.

Fig. 2 depicts BER versus normalized laser linewidth $\Delta \nu T_{\mathrm{s}}$ using $p_{\mathrm{a}}=2.65 \%$. The top plot shows results for $\sigma_{\mathrm{r}}^{2}=0$, corresponding to perfectly correlated phase noise across the spatial channels, whereas results for $\sigma_{\mathrm{r}}^{2}=\sigma_{\theta}^{2} / 1000$, i.e., when relative phase drifts are present, are shown in the bottom plot. In both cases, WD is used with $\beta=1$. For fully correlated channels, WD 


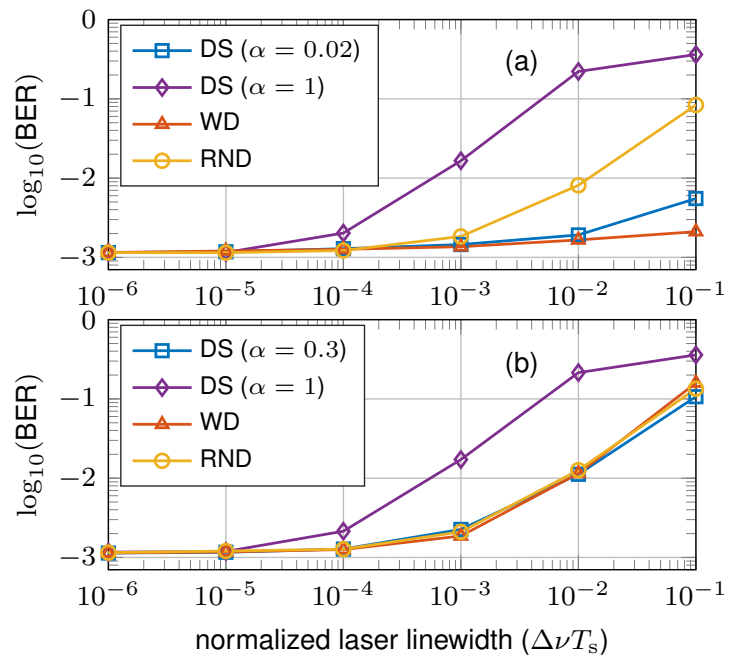

Fig. 2: Comparison of the pilot distributions, for fully correlated (a) and partially correlated (b) spatial channels.

yields the best performance overall for the tested pilot rate, maintaining a low penalty even at extreme normalized laser linewidths, whereas RND gives substantial penalty for high values of $\Delta \nu T_{\mathrm{S}}$. Moreover, DS with $\alpha=0.02$ performs slightly worse than WD, and for $\alpha=1$, which corresponds to identical pilot placements in all channels, DS yields the worst performance. As seen, the choice of distribution can affect the BER by a factor up to 170 . On the other hand, for partially correlated channels, i.e., when relative phase drifts are present, WD, RND, and DS with $\alpha=0.3$ perform similarly. As $\Delta \nu T_{\mathrm{s}}$ grows large, however, the performance of DS with $\alpha=0.3$ is superior. As before, DS with $\alpha=1$ gives the biggest BER penalty.

In general, the optimal value of $\alpha$ for DS, i.e., the value that yields the least BER penalty, depends on the ratio between the relative phase drift speed and the LPN speed, $\sigma_{\mathrm{r}}^{2} / \sigma_{\theta}^{2}$, as detailed in Fig. 3. To evaluate different ratios, the normalized laser linewidth is fixed while the core and polarization phase drift speeds are varied. When these phase drifts are orders of magnitude slower than the LPN, a low value of $\alpha$ is optimal, resulting in one channel having a much higher pilot rate than the remaining channels. This is intuitive given that the main impairment in this case is the LPN, which can be tracked more effectively if pilots are spaced closer to each other in time. Thus, having the majority of pilots closely packed in a single channel enables effective LPN tracking, and a small number of pilots in the remaining channels suffices to track the slower relative phase drifts. In contrast, as the speed of these phase drifts approaches that of the LPN, a higher value of $\alpha$ is more beneficial, producing a more similar pi-

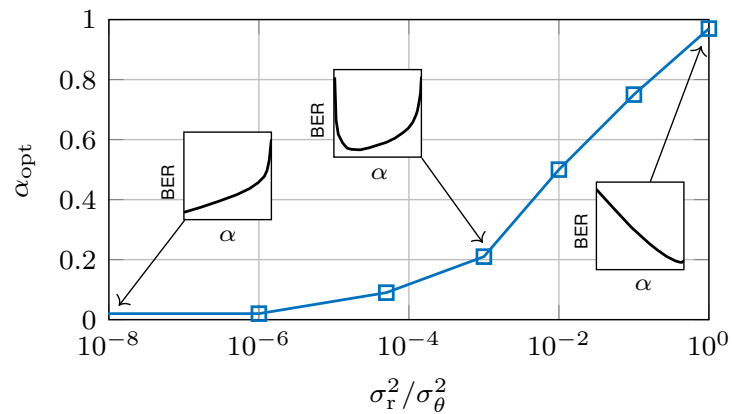

Fig. 3: The optimal value of $\alpha$ for DS, denoted by $\alpha_{\text {opt }}$, as a function of the ratio between $\sigma_{\mathrm{r}}^{2}$ and $\sigma_{\theta}^{2}$.

lot distribution across the channels. This is due to the relative phase drifts requiring more closely spaced pilots in each channel for effective tracking. Hence, assigning the majority of the available pilots to a single channel, corresponding to a low value of $\alpha$, is not optimal in that case.

\section{Conclusions}

Several types of pilot distributions were compared through Monte Carlo simulations in terms of the resulting BER performance for tracking of correlated phase noise in SDM transmission. It was shown that having identical pilot placements in all spatial channels is in general not optimal. Moreover, unless the phase noise is perfectly correlated across all channels, placing all available pilots in a single channel is also suboptimal. Rather, the distribution of pilots should depend on the amount of phase-noise correlation across the channels.

\section{Acknowledgments}

This work is supported by the Swedish Research Council (VR), Grants 2014-6138 and 2013-5642.

\section{References}

[1] B. J. Puttnam et al., "Investigating self-homodyne coherent detection in a 19 channel space-division-multiplexed transmission link," J. Lightwave Technol., Vol. 21, no. 2, p. 1561 (2013).

[2] A. F. Alfredsson et al., "Phase-noise compensation for spatial-division multiplexed transmission," Proc. OFC, Th4C.7, Los Angeles (2017)

[3] H. Minn et al., "Optimal training signals for MIMO OFDM channel estimation in the presence of frequency offset and phase noise," IEEE Trans. Commun., Vol. 54, no. 10, p. 1754 (2006).

[4] X. Ma et al., "Novel training symbol structure for transmitter IQ mismatch estimation for PDM coherent optical OFDM system," J. Lightwave Technol., Vol. 34, no. 22, p. 5183 (2016).

[5] X. Wu et al., "Impact of pilot positions on RF-Pilot based XPM compensation for CO-OFDM systems," Proc. ACP, AF2F.31, Beijing (2013).

[6] R. S. Luís et al., "Comparing inter-core skew fluctuations in multi-core and single-core fibers," Proc. CLEO, SM2L.5, San Jose (2015). 